Food Additives and Contaminants - Part A Chemistry, Analysis, Control, Exposure and Risk

Assessment, 2009, vol 26, issue 9, pp1298-1305

\title{
Modelling a two-dimensional spatial distribution of mycotoxin concentration in bulk commodities to design effective and efficient sample selection strategies.
}

\author{
M. Rivas Casado ${ }^{\mathrm{a}}$, D.J. Parsons ${ }^{\mathrm{a}}$, R.M. Weightman ${ }^{\mathrm{b}}$, N. Magan ${ }^{\mathrm{a}}$, S. Origgi ${ }^{\mathrm{c}}$ \\ ${ }^{a}$ Natural Resources Department, Cranfield University, Cranfield, Bedford, MK43 0AL, UK \\ ${ }^{\mathrm{b}}$ ADAS Centre for Sustainable Crop Management, ADAS UK Ltd., Boxworth, Cambridge, \\ CB23 4NN, UK \\ ${ }^{\mathrm{c}}$ Food Standards Agency, Aviation House, 125 Kingsway, London, WC2B 6NH, UK \\ *Corresponding author. Email: m.rivas-casado@cranfield.ac.uk
}

\begin{abstract}
Mycotoxins in agricultural commodities are a hazard to human and animal health. Their heterogeneous spatial distribution in bulk storage or transport makes it particularly difficult to design effective and efficient sampling plans. There has been considerable emphasis on identifying the different sources of uncertainty associated with mycotoxin concentration estimations, but much less on identifying the effect of the spatial location of the sampling points. This study used a two-dimensional statistical modelling approach to produce detailed information on appropriate sampling strategies for surveillance of mycotoxins in raw food commodities. The emphasis was on deoxynivalenol (DON) and ochratoxin A (OTA) in large lots of grain in storage or bulk transport. The aim was to simulate a range of plausible distributions of mycotoxins in grain from a set of parameters characterising the distributions. For this purpose, a model was developed to generate data sets which were repeatedly sampled to investigate the effect that sampling strategy and the number of incremental samples has on determining the statistical properties of mycotoxin concentration. Results showed that for most sample sizes, a regular grid proved to be more consistent and accurate in the estimation of the mean concentration of DON, which suggests that regular sampling strategies should be preferred to random sampling, where possible. For both strategies, the accuracy of the estimation of the mean concentration increased significantly up to sample sizes of 40-60 (depending on the simulation). The effect of sample size was small when it exceeded 60 points, which suggests that the maximum sample size required is of this order. Similar conclusions about the sample size apply to OTA, although the difference between regular and random sampling was small and probably negligible for most sample sizes.
\end{abstract}

\section{Keywords}

Geostatistical modelling; deoxynivalenol; ochratoxin A; bulk; cereals; sample size; sampling strategy.

\section{Introduction}

For consumer health to be effectively protected it is important that consumer exposure to natural toxic contaminants in food, such as mycotoxins, is minimised. The ability to obtain a representative sample for analysis, from a raw material or processed product, is critical as part of a prevention strategy. Previous studies have proved that designing sampling plans for mycotoxins is particularly problematical because of the heterogeneous distribution of these 
contaminants in bulk lots of different commodities (Stroka et al., 2004, Schatzki, 1995a and 1995 b and Jewers et al., 1988). Because mycotoxin sampling is time-consuming and expensive, a limited number of samples are taken to obtain an estimate of the mean concentration. Normal practice is to take several small quantities of the commodity, known as incremental samples, from different locations (European Commission, 2005). These are mixed together to form the aggregate sample, from which a portion is extracted for analysis. In this paper, the process of collecting several incremental samples is referred to as sampling, the set of discrete incremental samples is called the sample, and the number of incremental samples is the sample size.

Previous studies have looked at the complexities associated with the estimation of the mean mycotoxin concentration in bulk commodities. For example, Johansson et al. (2006) looked at the distribution of fumonisin and aflatoxin concentration in maize. They divided test maize samples into damaged kernels, whole kernels and other materials and found that toxins were concentrated in the poor quality components. As a result, they suggested that analyses in smaller samples of poor quality components of the sample provided a better prediction of the level of these mycotoxins in the bulk lot. Whitaker (2003) investigated the sources of error in the mycotoxin test procedure for aflatoxin in raw shelled peanuts and described it as the combination of number of errors including at sampling, sample preparation method, and analytical method stages. In his study, the sampling step was identified as the largest source of error. Other studies have looked at the coefficients of variation in relation to sampling, sample preparation and analysis for different aflatoxins (Whitaker, 2004, Whitaker 2006 and Whitaker and Wiser, 1969) and proved that the type of commodity affects the variation in percentage error at the sampling stage. Coker et al. (1995) reviewed the complexities associated with the design of sampling plans for different commodity types, sample composition, sample preparation method, analytical sample and batch acceptance level. Their review concluded that approximately 100 incremental samples are required to obtain a $10 \mathrm{~kg}$ representative aggregate sample in commodities composed of large particles. For oilseed cakes and meal, fifty incremental samples are sufficient to obtain a representative $5 \mathrm{~kg}$ aggregate sample. Miraglia et al. (2005) identified another source of error associated with the sampling steps. They divided the sampling procedure into primary and secondary sampling. The primary sampling focuses on determining where, why and when to take samples along the food chain. The secondary sampling aims at determining and establishing how samples should actually be collected, and more importantly how many samples to take to obtain a representative sample. Other studies have identified the difficulties of sampling the spatial aggregation of plant disease and have proposed different methodologies to address this limitation (Maanen and Xu, 2003). Macarthur et al. (2006) identified the need to design adequate sampling programmes that take into account the heterogeneity in the spatial distribution of mycotoxins so that decisions on acceptance/rejection of contaminated lots are more accurate. Whitaker (2006) discussed methods to reduce sampling error and stated that these methods should focus on defining the number of samples required to obtain a specific level of confidence on the mean mycotoxin concentration and on determining the location of the samples to be taken so the likelihood of detecting the mycotoxins is maximised.

There has been considerable emphasis on identifying the different sources of uncertainty associated with mycotoxin concentration estimations, but much less on developing the statistical basis for robust sampling plans that account for the spatial distribution of the mycotoxins in bulk commodities. Recent studies (Rivas Casado et al., 2009 and Parsons et al., 2007) have looked at the potential of geostatistics to characterise the spatial distribution of deoxynivalenol (DON) and ochratoxin A (OTA). Results showed that while no spatial structure could be identified for OTA due to its random occurrence, geostatistics was a useful tool to describe the spatial distribution of DON. Some studies have 
successfully looked at the application of geostatistics to characterise the spatial structure of Apergillus flavus in soil (Orum et al., 1999), fusarium crown and root rot in tomatoes (Rekah et al., 1999), plant pathogens in diseased plants (Chellemi et al., 1988), the downy mildew pathogen (Peronospora parasitica) in cabbage (Stein et al., 1994) and citrus tristeza virus (Gottwald et al. 1996).

This study used a two-dimensional statistical modelling approach to produce detailed information on sampling strategies for surveillance of mycotoxins in raw food commodities. The emphasis was on DON and OTA in large lots of grain.

\section{Method}

The model was developed using the $\mathrm{R}$ statistical programming language ( $\mathrm{R}$ Development Core Team, 2007) to investigate the effect that sample size and strategy have on determining the mean concentration of DON and OTA in bulk commodities. The method was divided in four sequential steps: generation of mycotoxin concentration data, spatial distribution of the generated data, repeated generation of samples and assessment of the performance of each sample size and strategy combination. The model was then run for a range of scenarios.

\section{Data generation}

Rivas Casado et al. (2009) analysed the spatial structure of DON and OTA in a $26 \mathrm{t}$ truck of wheat with a total of 100 sampled points, from a data set collected by Biselli et al. (2005). The results showed that the distribution of DON was best described by a log-normal distribution of mean $1342 \mu \mathrm{g} \mathrm{kg}^{-1}$ and standard deviation 340, and presented spatial structure. In contrast, OTA was best described by an exponential distribution with mean $0.57 \mu \mathrm{g} \mathrm{kg}^{-1}$ and standard deviation 1.13, and was randomly distributed in space. Rivas Casado et al. (2009) used geostatistical techniques to investigate the spatial distribution of DON in the truck in more detail.

Geostatistics analyses the spatial structure of a variable (in this case mycotoxin concentration) using the variogram, a graph that relates the distance between any two points, known as the lag distance, with their semivariance (Figure 1). Generally, points close together have more similar values than those farther apart, giving low semivariance, or conversely high autocorrelation, at short lag distances. Each variogram can be described by a set of parameters: the sill, the range and the nugget. The sill is the a priori variance, $\sigma^{2}$, of the process and is generally assumed to be equal to the variance of the population (Barnes, 1991). The range is the point at which the semivariance approaches the sill, so the autocorrelation between pairs of points becomes 0 , marking the limit of spatial dependence: points farther apart than this lag distance are spatially independent. The nugget is the semivariance at a lag distance of 0 , and identifies the measurement error and the variations that occur over lag distances less than the shortest sampling interval (Webster and Oliver, 2000). Geostatistical analysis is more effective when the variable has a normal (Gaussian) distribution. For this purpose, the data were log transformed (natural log) to meet the normality requirement. The results showed that the spatial structure of DON could be described by a variogram with range $4.35 \mathrm{~m}$, sill 0.07 and nugget 0.013 . The spatial structure defined by these parameters was assumed to be representative of DON in bulk commodities, in the absence of other data, and was therefore used as a reference condition for data generation.

Two different procedures for data generation were used based on the findings described by Rivas Casado et al. (2009): classical statistics for OTA and geostatistics for generation of DON concentration. The classical statistics procedure allowed the generation of random deviates of an exponential distribution with a density function: 


$$
f(x ; \lambda)=\left\{\begin{array}{rr}
\lambda e^{-\lambda x}, & x \geq 0 \\
0, & x<0
\end{array}\right.
$$

where $x$ is the concentration of the mycotoxin and $\lambda(\lambda>0)$ is the rate parameter estimated through maximum likelihood (Equation 2)

$$
\lambda=\frac{1}{\bar{x}}
$$

where $\bar{x}$ is the sample mean concentration. This only required the mean concentration of the mycotoxin as an input parameter for the generation of data.

The geostatistical procedure generated simulations of normal random fields for the given variogram parameters. This was implemented using the geoR and RandomFields packages from the $\mathrm{R}$ programming language ( $\mathrm{R}$ Development Core Team, 2007). This procedure required values for the sill, the range and the mean of the DON population to be generated. It was assumed that the data represented values that had been transformed to give a normal distribution, for example by a log transform, so the mean and sill should be those of the transformed data. The results were transformed back to the original units for interpretation. The procedure could not fully control the properties of the simulated population due to processes for random generation of values, so several were generated for each set of parameters, then the variogram for each was displayed and compared to the target variogram to select the one that best represented the target spatial distribution.

\section{Spatial distribution}

The sampled area was always represented by a 1 unit $\mathrm{x} 1$ unit square, which could be scaled to the required dimensions. The data values were generated on a regular grid of 2500 points $(50 \times 50)$ in this area according to the chosen spatial distribution. For OTA, the generated values were randomly assigned to the grid points. For DON, the values were distributed in the space according to the specified sill and range values. The grf function from the RandomFields package in $\mathrm{R}$ was used for this purpose. This function generates simulations of normal random fields and distributes them in a unit square accordingly to a given a set of variogram parameters. The generated data set was treated as the true representation of DON or OTA concentration in a bulk commodity from which samples were to be taken.

For each set of parameters for either the geostatistical or classical method, $M$ data sets were generated, where $M$ was usually set at 30 . The variability of the realisations generated was derived from these $M$ simulations (Figure 2).

\section{Repeated generation of samples}

The simulated distribution was sampled using different sample sizes and two sampling strategies, regular square grid and random, to derive statistics to allow them to be compared. Each sample size between 4 and 100 was then selected $R$ times per simulation, where $R$ was fixed at 30. For random sampling, the required number of values was drawn from randomly selected points, so all sample sizes between 4 and 100 were possible, and repetition used new random samples. For regular grids, the only sample sizes considered were those that formed squares, that is the nine regular grids with 2-10 points in each direction giving 4, 9, 16, 25, 36, 49, 64, 81 and 100 points. In this case, repeated samples from the same distribution were taken by changing the origin of the grid. There were thus a 
total of $87300(30 \times 97 \times 30)$ and $8100(30 \times 9 \times 30)$ automated results for the random and regular grid sampling strategies respectively (Figure 2).

\section{Assessment of the performance of sample size-strategy combinations}

Two statistics must be considered for the quantification of sample and population differences: accuracy and precision. Accuracy is measured by the bias $b$, which is the difference between the sample mean concentration $\hat{\mu}$ and the population mean concentration $\mu$. (Equation 3). If the mean of the sample is equal to the mean of the population, then $\hat{\mu}$ is said to be unbiased, otherwise it is said to be biased

$$
b=\hat{\mu}-\mu
$$

Precision is the degree of mutual agreement between all the points in the sample and is estimated by the standard deviation of the sample $\hat{\sigma}$ :

$$
\hat{\sigma}=\sqrt{\frac{\sum_{i=1}^{n}\left(x_{i}-\hat{\mu}\right)^{2}}{n-1}}
$$

The performance of each of the combinations of sample size and sampling strategy was assessed using the Root Mean Squared Error (Equation 5). This statistic combines accuracy and precision. The smaller the RMSE, the better the estimation of the sample mean. The RMSE has the advantage of having the same units as the variable under study $\left(\mu \mathrm{g} \mathrm{kg}^{-1}\right)$.

$$
R M S E=\sqrt{\hat{\sigma}^{2}+b^{2}}
$$

A set of $R M S E_{i n r}$ where $i$ is the simulation number $(1 \leq i \leq 30), n$ is the sample size $(4 \leq n \leq 100)$ and $r$ is the repetition $(1 \leq r \leq 30)$ was obtained by repeatedly calculating RMSE values for a specific sample size. The distribution of RMSE values is approximately normal when more than 30 repetitions are calculated (Cochran, 1953) and therefore, confidence intervals can be calculated. The size of the confidence interval for each sample size and strategy was calculated by multiplying the standard error of the 30 RMSE values by the standard normal value $c$ for a specified probability level. To model the effect that the sample size had on the RMSE, a locally weighted regression smoothing (LOESS) function (Higgins, 2004) was fitted to the RMSE confidence interval with the sample size as the independent variable.

\section{Level of contamination}

The analysis was repeated using three different means, for both DON and OTA, to have a representative result for a range of concentrations. The selected concentrations were below, close and above the maximum level of DON $\left(1250 \mu \mathrm{g} \mathrm{kg}^{-1}\right)$ and OTA $\left(5 \mu \mathrm{g} \mathrm{kg}^{-1}\right)$ set by the European Commission (European Commission, 2006). For DON the model was run with log-normal DON data sets with mean $6.43,7.14$ and 7.82 , that is $\log _{e} 625 \mu \mathrm{g} \mathrm{kg}^{-1}$, $\log _{\mathrm{e}} 1261 \mu \mathrm{g} \mathrm{kg}^{-1}$ and $\log _{\mathrm{e}} 2490 \mu \mathrm{g} \mathrm{kg}^{-1}$, respectively. For OTA, the model was run with concentrations $0.6 \mu \mathrm{g} \mathrm{kg}^{-1}$ (found in the data from Biselli et al. 2005), $5 \mu \mathrm{g} \mathrm{kg}^{-1}$ and $10 \mu \mathrm{g} \mathrm{kg}^{-1}$. The spatial structure parameters found in the data from Biselli et al. were used as reference values to generate DON data for any of the three mean concentrations considered. The variogram parameters used were sill 0.07 , range $4 \mathrm{~m}$, and nugget 0 . 
A total of 30 simulations $(M=30)$ of log-normal DON data sets with mean 7.14 and 30 simulations of exponential OTA data sets with mean $0.6 \mu \mathrm{g} \mathrm{kg}^{-1}$ were generated to assess the reliability of the methods for data generation. The results showed that there was little variation between simulation, so subsequently only one was run for each set of parameters.

\section{Results}

For DON, the results for the assessment of the reliability of the geostatistical method for data generation showed that the approximations to the requested population mean and standard deviation were close to the target values: for the 30 simulations the average of the population mean was 7.15 , which was close to the 7.14 target mean. The maximum and minimum population mean obtained were 7.26 and 7.00 , respectively. The standard deviation $(\sigma=0.27)$ obtained was also very close to the target value $(\sigma=0.23)$, this being determined by the sill of the spatial structure.

The simulated DON spatial structure showed that the nugget and the sill were close to the required values. The mean sill was 0.069 with a standard deviation of 0.0068 which was very close to the 0.07 target. This was consistent with the results obtained for the standard deviation of the population. The mean nugget was 0.0010 with a standard deviation of 0.0037 which was also very close to the 0 target nugget. The values of the range did not accurately adjust to the target value. The mean range obtained for the 30 repetitions was 0.165 units with a standard deviation of 0.056 , which differed considerably from the target value of 0.4 units.

For OTA and a target mean of $0.6 \mu \mathrm{g} \mathrm{kg}^{-1}$, the thirty simulations had a mean value of $0.602 \mu \mathrm{g} \mathrm{kg}^{-1}$ and a standard deviation of 0.012 . This showed that there was little variation between generated data sets for the same target value.

For both cases, the statistics derived by repeated sampling from different simulations with the same parameters differed little between simulations. As noted above, it was concluded that it was sufficient to generate one simulated population for each set of parameters.

The RMSE changed in proportion to the mean concentration. For example, for the simulation of DON with mean concentration of $625 \mu \mathrm{g} \mathrm{kg}^{-1}$ and for different samples, the RMSE was $90-300 \mu \mathrm{g} \mathrm{kg}^{-1}$ (14-48\% of the mean) and for the simulation with mean concentration of $2490 \mu \mathrm{g} \mathrm{kg}^{-1}$ it was $300-1100 \mu \mathrm{g} \mathrm{kg}^{-1}$ (12-44\%). Similar results were seen for OTA.

The general pattern observed for the majority of the LOESS equations fitted showed a decrease in the slope of the curve at 40-60 samples (Figure 3), beyond which the RMSE did not decrease as significantly for each increment in the sample size. The RMSE is made up of the sample standard deviation and the bias. The bias should tend to 0 when increasing the sample size and therefore the main contribution to the RMSE for large samples is the standard deviation of the sample. For sample sizes close to the population size, the RMSE would be very close to the standard deviation of the population and no further gain in accuracy could be obtained. This point was reached at 40-60 samples.

For DON, the graphs of RMSE $+95 \%$ confidence interval (Figure 4a) showed that the results of regular grid sampling were more consistent than random sampling. For a given sample size, the RMSE for random sampling was sometimes as low as or lower than that for regular grid sampling, but in most cases it was higher. This is probably the result of bias introduced by clusters of relatively closely spaced, and therefore correlated, points. This suggests that it is better to use regular grid sampling strategies when spatial structure is expected for the mycotoxin concentration. For OTA (Figure 4b), the difference between the strategies was small. The scatter-plots for the RMSE $+95 \%$ confidence interval showed 
overlapping results for the two methods. In general, for the OTA data sets the difference between the two sampling strategies was negligible.

\section{Discussion}

The assumptions made in the model and its limitations, particularly the distributions used, must be considered when interpreting the results and should be verified in practice.

The model was reliable at generating data sets with the target DON and OTA mean concentration. However, in some cases the model failed to reproduce the target spatial structure for DON. When the maximum range value generated was below the target range, a less smooth spatial distribution than intended was generated. In general this would be expected to reduce the differences between random and regular sampling, and to increase the bias. Despite this, clear differences were found between the two sampling methods. If the intention in future was to simulate a particular observed distribution, this could be done by increasing the target value of the range until the result from the simulated data matched the observation. In this case the aim was to examine a range of possible distributions. Consistent results were obtained across all mean concentrations, and contrasts were found between random and regular sampling.

For both strategies and both mycotoxins, the accuracy of the estimation of the mean concentration increased significantly up to sample sizes of 40-60 (depending on the simulation). The effect of sample size was small when it exceeded 60 points, which suggests that the maximum sample size required is of this order. European Commission regulations specify 60 incremental samples from grain lots of 10-20 t and 100 incremental samples from lots over $20 \mathrm{t}$ (European Commission, 2005). The model results show that these would be adequate in the cases simulated. On this basis, there is no evidence to recommend either a reduction or an increase in the number of incremental samples. These results are also consistent with the findings of Coker et al. (1995) that 50-100 samples are required for different aggregate sample masses.

The model was designed to simulate lots of the order of 10-100 t. There is no evidence on the spatial structure at smaller or larger scales so the results should not be extended at these scales without further data collection and analysis.

The samples used in the simulation are idealised: each is the exact value at a single point, and the mean is calculated precisely. In practice each incremental sample takes a small mass of grain, these are mixed and the concentration is measured in a subsample. Thus there are two additional sources of error: the difference between finite incremental samples and point measurements, and the difference between the mean of the idealised samples and the concentration measured from the aggregated sample. The effects of mixing and subsampling have been investigated by Whitaker $(2004,2006)$, and should be considered when examining the total uncertainty. The mass of an incremental sample is normally $0.1-1.0 \mathrm{~kg}$, which implies that the dimensions are of the order of $0.1 \mathrm{~m}$. This is small compared to the scale at which the model is intended to operate. It is therefore reasonable to treat the incremental samples as point measurements.

The random sampling method in the model is truly random: all points are equally likely to be sampled, independent of which others have been selected. In practice, when someone samples from a bed of grain, they are unlikely to take two samples very close together, so real sampling behaviour is likely to be intermediate between random sampling and regular grid sampling.

The model was based on a single set of data for DON and OTA. Further research would be needed before drawing more general conclusions. 
The model does not consider the variation in mycotoxin concentration with depth, but only in two horizontal dimensions, because no data were available. Indeed, sampling using a conventional sample spear aggregates over the depth from one point on the surface. The data used to develop the model came from this type of sampling, so it is intended to be an empirically based representation of normal practice. Recent works on geostatistical science provide a methodology to develop 3D geostatistical models (Culshaw, 2005 and Stavropoulou et al., 2007) and could be applied to mycotoxin concentration, if suitable data sets were collected.

\section{Conclusions}

The model focused on the effect that the sampling protocol has on determining the statistical properties of mycotoxin concentration. The conclusions drawn in this study must be understood as preliminary outcomes of the model. The assumptions made in the development of the model and its limitations must be considered when interpreting the conclusions.

This study showed that the accuracy of the estimation of the mean concentration increased significantly up to sample sizes of 40-60, which is consistent with the number of incremental samples taken from bulk lots under EU regulations. This applied to both spatially structured and randomly distributed data across the range of mean concentrations used.

A regular grid proved to be more consistent and accurate in the estimation of the mean concentration where there was spatial structure (DON). The difference between the two sampling strategies was negligible for randomly distributed data (OTA). This suggests that regular sampling strategies should be preferred to random sampling, where possible.

\section{Acknowledgements}

This research was funded by the Food Standards Agency Project CO3055. We wish to thank Dr. Scarlett Bisselli (EUROFINS, Hamburg, Germany) for giving permission to use the data on which the model is based.

\section{References}

Barnes RJ. 1991. The variogram sill and the sample variance. Math Geol. 23(4): 673-678

Biselli S, Bruer J, Persin M, Schuh M, Syben M. 2005. Investigation of variability associated with testing lots of wheat kernels for deoxynivalenol and ochratoxin A (case study truck). Paper presented at the World Mycotoxin Forum 2005. Proceedings of the $3^{\text {rd }}$ World Mycotoxin Forum; Noordwijk, The Netherlands.

Chellemi DO, Rohrbach KG, Yost RS, Sonoda RM. 1988. Analysis of the spatial pattern of plant-pathogens and diseased plants using geostatistics. Phytopathology. 78(2): 221226.

Cochran WG. 1953. Sampling Techniques, $2^{\text {nd }}$ Ed. New York: John Wiley and Sons

Coker RD, Nagler MJ, Blunden G, Sharkey AJ, Defize PR, Derksan GB, Whitaker TB. 1995. Design of sampling plan for mycotoxins in foods and feeds. Nat Toxins 3: 257-264.

Culshaw, M.G. 2005. From concept toward reality: developing the attributed 3D geological model of the shallow subsurface. Q J Eng Geol Hydroge. 38: 231-284. 
European Commission. 2005. Commission Directive 2005/38/EC of 6 June 2005. Laying down the sampling methods and the methods of analysis for the official control of the levels of Fusarium toxins in foodstuffs. Off J Eur Union. L143: 18-26.

European Commission. 2006. Commission Regulation 1881/2006 of 19 December 2006. Setting maximum levels for certain contaminants in foodstuffs. Off J Eur Union. L364: 5-24.

Gottwald TR, Cambra M, Moreno P, Camarasa, E, Piquer J. 1996. Spatial and temporal analyses of citrus tristeza virus in eastern Spain. Phytopathology. 86(1): 45-55.

Higgins JJ. 2004. Introduction to modern nonparametric statistics. Duxbury advanced series. Pacific Grove, CA: Brooks/Cole

Jewers K, Bradburn N, Sharkey AJ. 1988. Aflatoxin distribution studies on a 4 tonne batch of maize. Int Biodeterior. 24(4-5): 393-398.

Johansson AS, Whitaker TB, Hagler WM JR, Bowman T, Slate AB, Payne G. 2006. Predicting aflatoxin and fumonisin in shelled corn lots using poor-quality grade components. J Assoc Off Ana Chem. 89: 433-440.

Maanen A, Xu XM. 2003. Modelling plant disease epidemics. Eur J Plant Pathol. 109: 669682.

Macarthur R, Macdonald S, Brereton P, Murray A. 2006. Statistical modelling as an aid to the design of retail sampling plans for mycotoxins in food. Food Addit Contam. 23 (1): 84-92.

Miraglia M, De Santis B, Minardi V, Debegnach F, Brera C. 2005. The role of sampling in mycotoxin contamination: an holistic view. Food Addit Contam. A, 22(1 S1): 31-36.

Orum TV, Bigelow DM, Cotty PJ, Nelson MR. 1999. Using predictions based on geostatistics to monitor trends in Aspergillus flavus strain composition. Phytopathology. 89(9): 761-769.

Parsons D, Rivas Casado M, Magan N, Dyer C, Weightman R. 2007. Development of representative sampling plans for mycotoxins in foods using distribution modelling. Final Report to the UK Food Standards Agency on Project CO3055. Wolverhampton: ADAS UK Ltd.

R Development Core Team. 2007. R: A Language and Environment for Statistical Computing. Vienna: R Foundation for Statistical Computing. http://www.Rproject.org .

Rekah Y, Shtienberg D, Katan J. 1999. Spatial distribution and temporal development of fusarium crown and root rot of tomato and pathogen dissemination in field soil. Phytopathology. 89(9): 831-839.

Rivas Casado M, Parsons DJ, Weightman RM, Magan N, Origgi S. 2009 submitted Geostatistical analysis of the spatial distribution of mycotoxin concentration in bulk cereals. Food Addit Contam. 26: 867-873

Schatzki TF. 1995a. Distribution of aflatoxin in pistachios. 1. Lot distributions. J Agr Food Chem. 43(6): 1561-1565.

Schatzki TF. 1995b. Distribution of aflatoxin in Pistachios. 2. Distribution in freshly harvested pistachios. J Agr Food Chem. 43(6): 1566-1569. 
Stavropoulou M, Exadaktylos G, Saratsis G. 2007. A combined three-dimensional geologicalgeostatistical-numerical model of underground excavations in rock. Rock Mech Rock Eng. 40 (3): 213-243.

Stein A, Kocks CG, Zadocks JC, Frinking HD, Ruissen MA, Myers DE. 1994. A geostatistical analysis of the spatio-temporal development of downy mildew epidemics in cabbage. Phytopathology. 84(10): 1227-1238.

Stroka J, Spanjer M, Buechler S, Barel S, Kos G, Anklam E. 2004 Novel sampling methods for the analysis of mycotoxins and the combination with spectroscopic methods for the rapid evaluation of deoxynivalenol contamination. Toxicol Lett. 153(1): 99-107.

Webster R, Oliver MA. 2000. Geostatistics for environmental scientists. Chichester: John Wiley and Sons, Ltd.

Whitaker TB, Wiser EH. 1969. Theoretical investigations into the accuracy of sampling shelled peanuts for aflatoxin. J Am Oil Chem Soc. 46(7): 377-379.

Whitaker TB. 2003. Standardisation of mycotoxin sampling procedures: an urgent necessity. Food Control. 14 (4): 233-237.

Whitaker TB. 2004. Mycotoxins in food: detection and control (eds. Magan N, Olsen M). Cambridge: Woodhead Publishing Ltd. Chapter 4, Sampling for mycotoxins: pp 6987.

Whitaker TB. 2006. Sampling foods for mycotoxins. Food Addit Contam. 23(1): 50-61. 


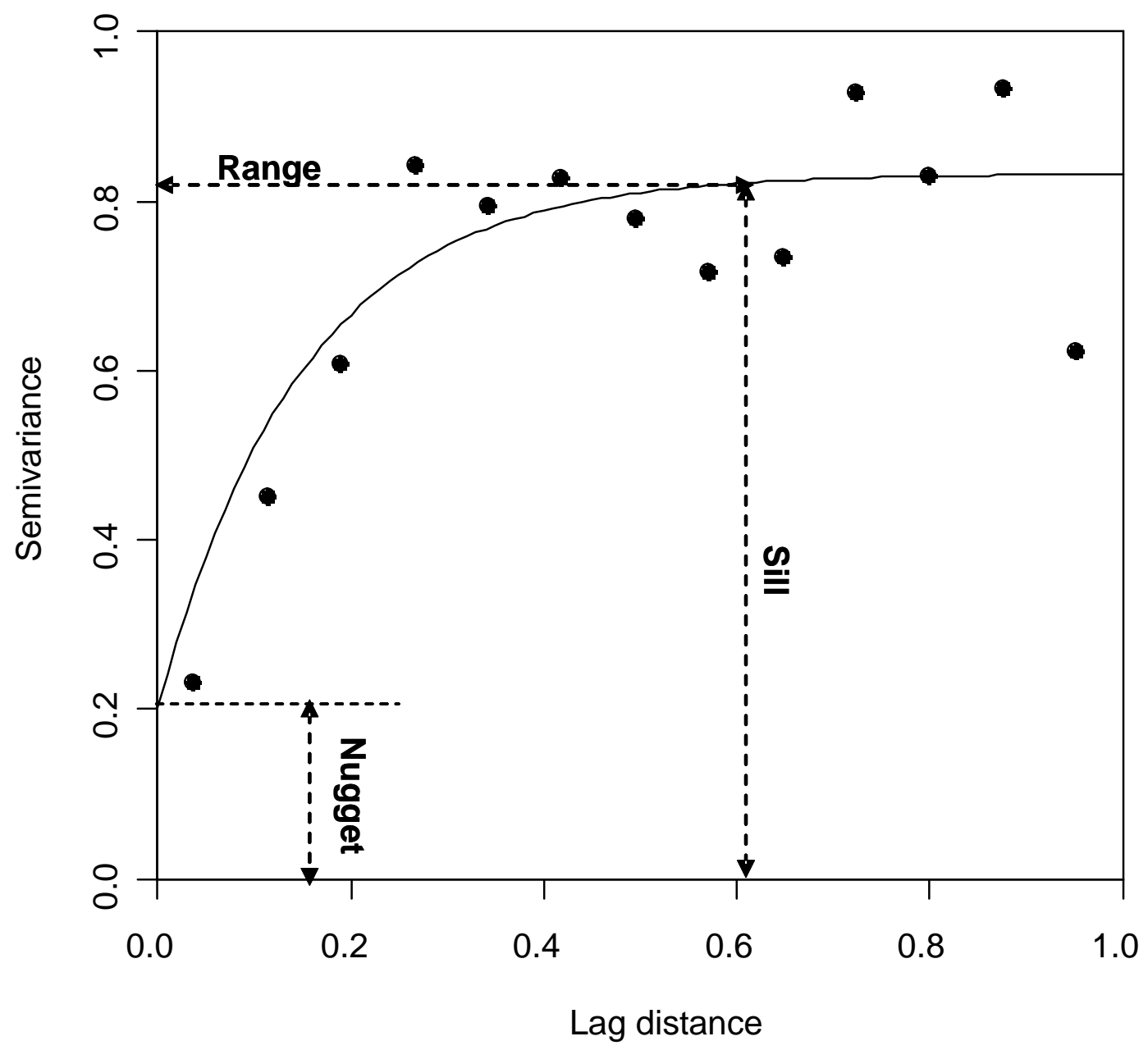

Figure 1. Example of a variogram function showing the sill, range and nugget parameters. 


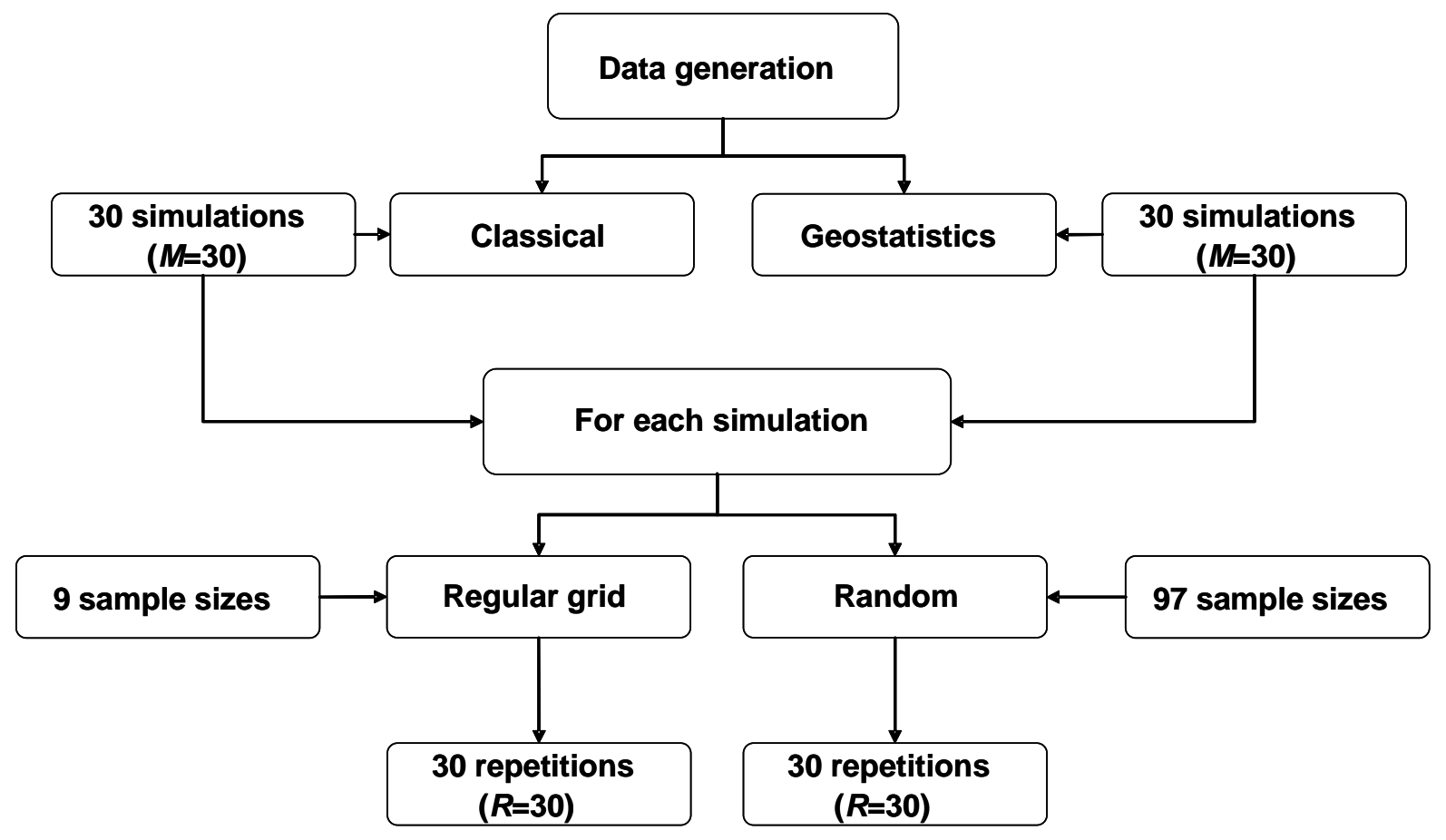

Figure 2. The iterative process followed by the model. 

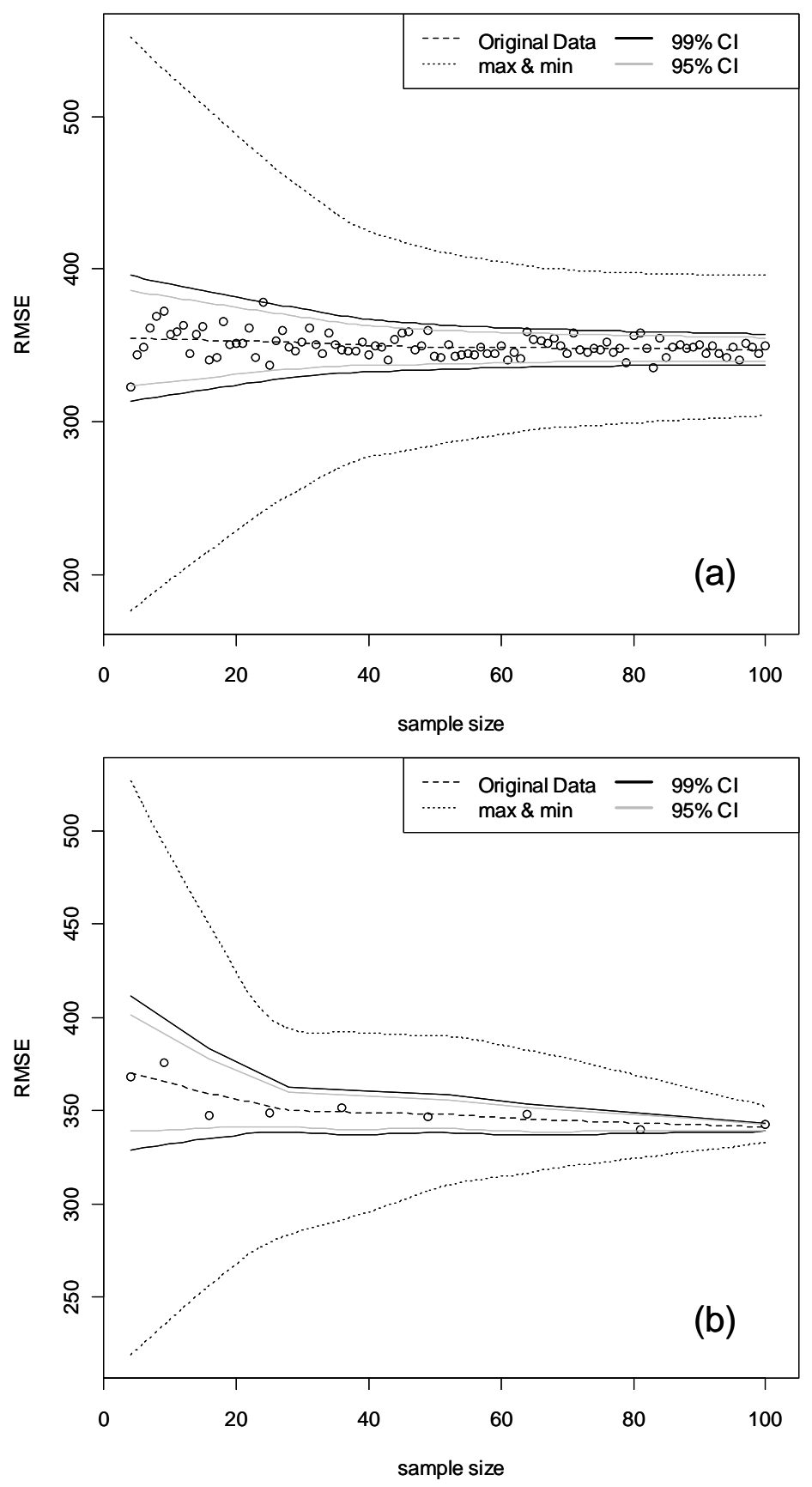

Figure 3. Example of the changes in the value of RMSE $\left(\mu \mathrm{g} \mathrm{kg}^{-1}\right)$ from sample size 4-100 observed for DON for random (a) and regular (b) sampling strategies. The model was run to simulate a bulk commodity with mean DON concentration $7.14\left(\log _{\mathrm{e}} 1260 \mu \mathrm{g} \mathrm{kg}^{-1}\right)$ and a spatial distribution described by a variogram with range $4 \mathrm{~m}$, sill 0.07 and nugget 0 . The final simulated values were mean 6.5, range 3.66, sill 0.067 and nugget 0.0091 . 

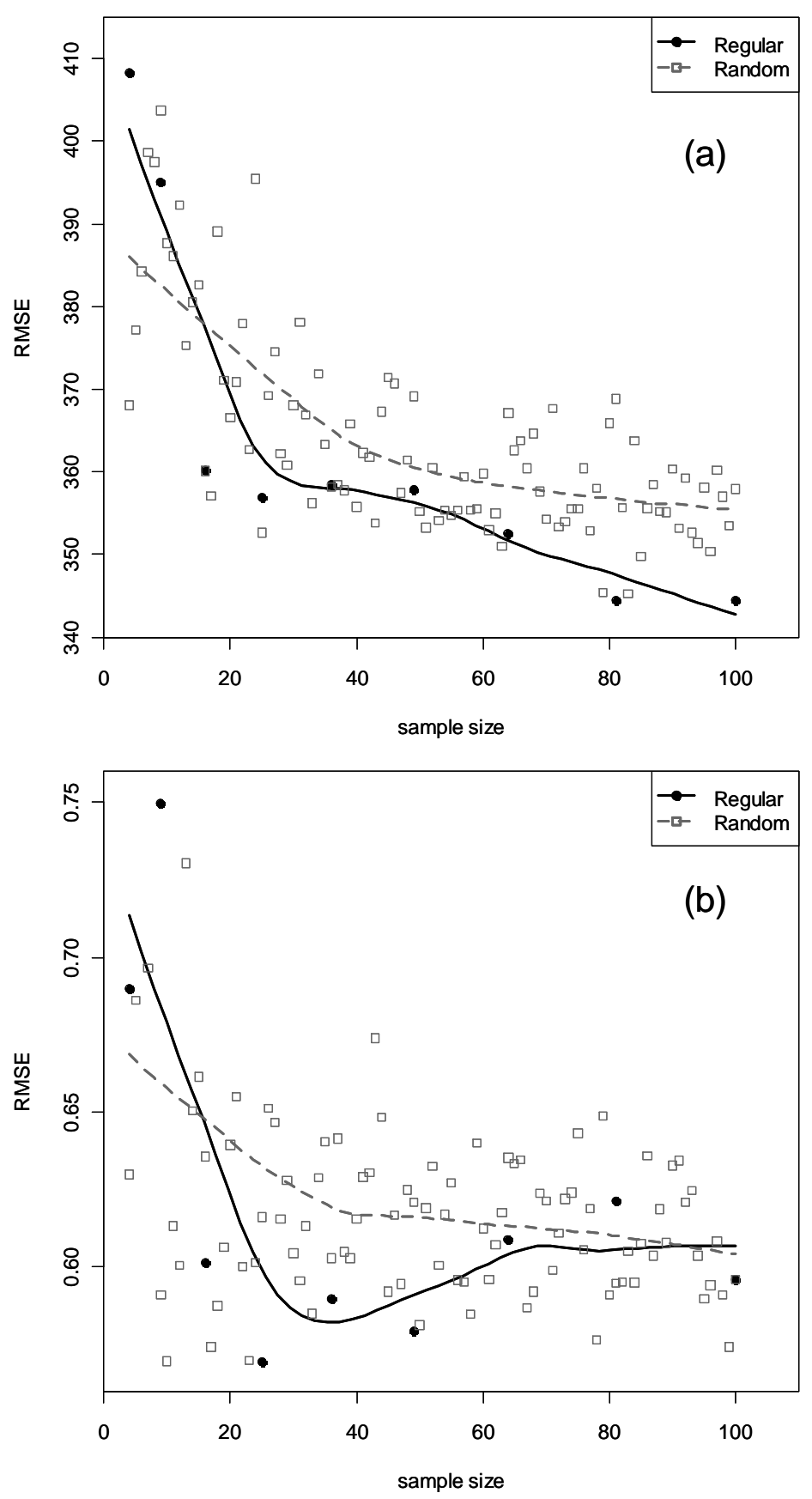

Figure 4. Example of the changes in the value of the RMSE plus the upper $95 \%$ confidence interval $\left(\mu \mathrm{g} \mathrm{kg}^{-1}\right)$ from sample size 4-100 obtained for the random and regular grid sampling strategies. The model was run to simulate a bulk commodity with (a) a mean DON concentration of $7.14\left(\log _{\mathrm{e}} 1260 \mu \mathrm{g} \mathrm{kg}^{-1}\right)$ and a spatial distribution described by a variogram with range $4 \mathrm{~m}$, sill 0.07 and nugget 0 ; and (b) a mean OTA concentration of $0.6 \mu \mathrm{g} \mathrm{kg}^{-1}$ and random spatial distribution. The final simulated values were mean 6.5, range 3.66, sill 0.067 and nugget 0.0091 for DON and mean 0.577 for OTA. 